\title{
Bilinear and nonlinear control algorithms for a DC/DC converter for Multi-Terminal HVDC networks *
}

\author{
M. Jiménez Carrizosa* G. Damm** A. Benchaib *** \\ P. Alou ${ }^{* * *}$ M. Netto ${ }^{\dagger}$ and F. Lamnabhi-Lagarrigue* \\ * Laboratoire des signaux et systèmes, Suplec, 3, Rue Joliot-Curie, \\ 91192, Gif-sur-Yvette, France (email: Miguel.Jimenez@lss.supelec.fr) \\ ** Laboratoire IBISC, Bâtiment Pelvoux 2, 40, Rue du Pelvoux CE1455 \\ Courcouronnes, 91020, Evry, France (email: gilney.damm@ibisc.fr) \\ *** ALSTOM GRID, 102 Avenue de Paris, 91300, Massy, France \\ (email: abdelkrim.benchaib@alstom.com) \\ **** Escuela Técnica Superior de Ingenieros Industriales, 2 José \\ Gutiérrez Abascal, 28006, Madrid, Spain (e-mail: pedro.alou@upm.es) \\ $\dagger$ IFSTTAR, 14, Route de la Minire, Bat 824, 78000, Versailles, \\ France (e-mail: mariana.netto@ifsttar.fr)
}

\begin{abstract}
This paper presents the modeling and control of a multilevel DC/DC bidirectional converter suitable for medium voltage and medium power applications, with a special interest in renewable applications. The multilevel converter is based on Dual Active Bridge (DAB) and its average dynamic model. Two different control strategies are addressed in this paper in order to achieve the overall control of the DC/DC converter: a bilinear systems' control based on quadratic feedback control, and a nonlinear control based in Lyapunov theory. System's controllability and stability are studied, in particular by the analysis of zero dynamics. The performance of both controllers are illustrated by computer simulations, and the concluding remarks analyze theirs characteristics and draw some comparisons.
\end{abstract}

Keywords: Control of bilinear systems, nonlinear control, DC/DC converters, Multi-Terminal HVDC.

\section{INTRODUCTION}

The increasing development of electricity needs has brought current $\mathrm{AC}$ grids close to their saturation. In the same time, the increase of renewable sources, with their intermittent and variable nature, and their ever increasing penetration in the classic $\mathrm{AC}$ power networks makes this problem even more delicate. It is in this context where Multi-Terminal High Voltage Direct Current (MT-HVDC) grids have gained importance with respect to $\mathrm{AC}$ grids. Some of their advantages is that they do not have reactive power, so their transmission capacities are increased, they also produce fewer losses than $\mathrm{AC}$ grids, the weight and cost of components are smaller (fewer cables), and the efficiency is higher (see Arrillaga et al. (2007); Asplund (2007)). It is clear than in MT-HVDC the DC/DC converters play a decisive role, since energy is generated at low voltages and highly efficient devices are needed to increase these voltages to higher values or to interconnect DC Grids at different voltage levels.

The control of DC/DC converters has been highly studied over the last decades, but especially in low voltage and low power applications. However the same philosophy of these techniques may be adapted for high or medium

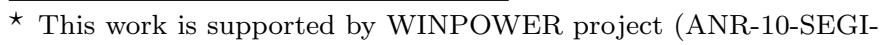
016). voltage and power applications. These techniques can be addressed from different viewpoints. One of them is to use the average model in each switching cycle of the control variables (see Sira-Ramirez et al. (1994)). To achieve this average model, pulse width modulation (PWM) is commonly used. Whatever the control objective, the average model of DC/DC converters via PWM have a structure of bilinear system.

A system is called bilinear if it is described by linear differential equations in which the control inputs appear as coefficients. The study of bilinear systems began in the 60 's as a gateway between the linear and nonlinear systems. A large amount of definitions and properties have been formulated until our days with respect to bilinear systems and their control, see: Mohler (1973, 1991); Landau (1979); Elliot (2009). However there are not yet general results to study their stability and controllability as in the case of linear systems. There exists only global proofs in the case of $2^{\text {nd }}$ order bilinear systems (see Koditschek and Narendra (1985); Barras et al. (1996); Ayala et al. (2009); Koditschek and Narendra (1983)). Due to this fact, it is very usual to treat bilinear systems as nonlinear systems and apply all the results of nonlinear control, since the theory of nonlinear control has global results on stability and controllability (see Isidori (1995); Khalil (2002)). In this paper, both a bilinear controller and a nonlinear 
controller based on Lyapunov theory are designed and compared.

The paper is outlined as follows: Section II presents the modeling of the dual active bridge which is the considered converter. Section III presents the control of a single DAB converter cell, by either a bilinear and a nonlinear controls. Section IV presents the simulations of these controllers. In Section V a comparison of both controllers is shown. Finally, in Section VI the conclusions are explained.

\section{2. $\mathrm{DAB}$}

The dual active bridge, DAB (see figure 1), is a widely used DC/DC converter for high voltage (more than $2 \mathrm{kV}$ ) or medium (around 1-2 kV) applications. It is composed of two full bridge converters, each one connected to one side of the transformer, which provides galvanic insulation necessary to protect against unforeseen circumstances (see G.Ortiz et al. (2010)). An inductance L, which is used as an energy transfer device, is placed in series with the transformer. Usually in each bridge it can be used either two phases (high voltage applications) as three phases (medium voltage). In this work we have chosen the last, in order to apply the $d q$ transformation. DAB is shown in figure 1, the objective of this paper is to implement a control algorithm taking into account its three state variables. Thereby improving the performance of the control compared to previous results in the literature as Jiménez et al. (2013).

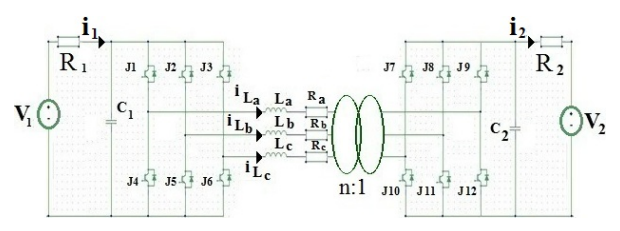

Fig. 1. Dual Active Bridge.

\subsection{Averaged dynamic model}

Thanks to the well known technique Pulse Width Modulation (PWM), it is possible to obtain an averaged dynamic model from the system shown in figure 1 which is shown in figure 2, notice that all devices are in the primary side of the transformer, where $n$ is the transformer ratio. This way of proceeding, in order to achieve an average model, is standard, as for example in Barragán-Villarejo et al. (2012). It must be emphasized that using this technique, it appears higher switching losses, so its use for high voltage levels is not recommended.

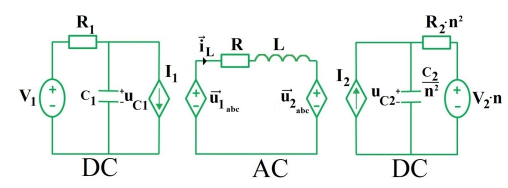

Fig. 2. DAB average model.

The converter can then be modeled as in Figure 2, where $u_{1 a}=u_{C 1} m_{1} \cos (\omega t+\delta)$ and $u_{2 a}=u_{C 2} m_{2} \cos (\omega t)$, with $0<m_{1}<1,0<m_{2}<1$ and $\delta$ is the phase for AC left side. So using the $d q$ transformation (see Park (1929)), we obtain that:

$$
\left\{\begin{array}{rlrl}
u_{1 d} & =\sqrt{\frac{3}{2}} u_{C 1} m_{1 d} & -1<m_{1 d}=m_{1} \cos (\delta)<1 \\
u_{1 q} & =-\sqrt{\frac{3}{2}} u_{C 1} m_{1 q} & -1<m_{1 q}=m_{1} \sin (\delta)<1 \\
u_{2 d}=\sqrt{\frac{3}{2}} u_{C 1} m_{2 d} & 0<m_{2 d}=m_{2}<1 \\
u_{2 q}=0 &
\end{array}\right.
$$

where $u_{1 d}$ and $u_{1 q}$, are the $d q$ voltage components of the three phases voltage system $u_{1 a}, u_{1 b}$, and $u_{1 c}$ (AC left side), and in analogous form for $u_{2 d}$ and $u_{2 q}$ (AC right side).

It should be noted that $u_{2 q}=0$, and this is due to our choice of the voltage phase in the right side of the converter was zero. This is in concordance with the real behavior of the system, because when we work in $d q$ frame, a phase lock loop (PLL) is necessary in order to generate a signal whose phase is related to the phase of an input signal (see Mendel et al. (2009)). Usually in electronic devices this technique is used to detect the frequency of the voltage and current and to detect the phase between them. So a voltage reference is needed, and if we select voltage $u_{2}$ as reference, its phase is zero, and consequently $m_{2 q}=0$, and therefore $u_{2 q}=0$.

On the other hand, and taking into account the power equalities $u_{C 1} I_{1}=u_{1 a} i_{L a}+u_{1 b} i_{L b}+u_{1 c} i_{L c}$ and $u_{C 2} I_{2}=$ $u_{2 a} i_{L a}+u_{2 b} i_{L b}+u_{2 c} i_{2 c}$, and carry out the Park's transformation we obtain that:

$$
\left\{\begin{array}{l}
I_{1}=\sqrt{\frac{3}{2}}\left(m_{1 d} i_{L d}+m_{1 q} i_{L q}\right) \\
I_{2}=\sqrt{\frac{3}{2}} m_{2 d} i_{L d}
\end{array}\right.
$$

The system shown in figure 2 can then be described by:

$$
\left\{\begin{array}{l}
\frac{d}{d t} i_{L d}=\frac{1}{L}\left(-R \cdot i_{L d}+\omega \cdot L \cdot i_{L q}+\sqrt{\frac{3}{2}}\left(m_{1 d} \cdot u_{c 1}-m_{2 d} \cdot u_{c 2}\right)\right) \\
\frac{d}{d t} i_{L q}=\frac{1}{L}\left(-\omega \cdot L \cdot i_{L d}-R \cdot i_{L q}+\sqrt{\frac{3}{2}} m_{1 q} \cdot u_{c 1}\right) \\
\frac{d}{d t} u_{C 1}=-\frac{1}{C_{1}}\left(\sqrt{\frac{3}{2}}\left(m_{1 d} \cdot i_{L d}+m_{1 q} \cdot i_{L q}\right)-\frac{V_{1}-u_{C 1}}{R_{1}}\right) \\
\frac{d}{d t} u_{C 2}=\frac{n^{2}}{C_{2}}\left(\sqrt{\frac{3}{2}} m_{2 d} i_{L d}-\frac{u_{C 2}-V_{2} \cdot n}{R_{2} \cdot n^{2}}\right)
\end{array}\right.
$$

where $i_{L d}, i_{L q}, u_{C 1}, u_{C 2}$ are state variables, $m_{1 d}, m_{2 d}, m_{1 q}$ are control variables and $V_{1}, V_{2}$ are external variables.

The systems equations shown in (3) may be represented by:

$$
\frac{d}{d t} \mathbf{x}=\mathbf{A} \mathbf{x}+\sum_{i=1}^{5} u_{i} \mathbf{B}_{i} \mathbf{x}+\mathbf{C u}
$$

where

$$
\mathbf{A}=\left[\begin{array}{cccc}
-\frac{R}{L} & \omega & 0 & 0 \\
-\omega & -\frac{R}{L} & 0 & 0 \\
0 & 0 & -\frac{1}{R_{1} C_{1}} & 0 \\
0 & 0 & 0 & -\frac{1}{R_{2} C_{2}}
\end{array}\right] ; \quad \mathbf{B}_{1}=\sqrt{\frac{3}{2}}\left[\begin{array}{cccc}
0 & 0 & \frac{1}{L} & 0 \\
0 & 0 & 0 & 0 \\
-\frac{1}{C_{1}} & 0 & 0 & 0 \\
0 & 0 & 0 & 0
\end{array}\right]
$$




$$
\begin{gathered}
\mathbf{B}_{2}=\sqrt{\frac{3}{2}}\left[\begin{array}{cccc}
0 & 0 & 0 & -\frac{1}{L} \\
0 & 0 & 0 & 0 \\
0 & 0 & 0 & 0 \\
\frac{n^{2}}{C_{2}} & 0 & 0 & 0
\end{array}\right] ; \quad \mathbf{B}_{3}=\sqrt{\frac{3}{2}}\left[\begin{array}{cccc}
0 & 0 & 0 & 0 \\
0 & 0 & \frac{1}{L} & 0 \\
0 & -\frac{1}{C_{1}} & 0 & 0 \\
0 & 0 & 0 & 0
\end{array}\right] \\
\mathbf{B}_{4}=\mathbf{B}_{5}=\mathbf{0} \\
\mathbf{C}=\left[\begin{array}{lllll}
0 & 0 & 0 & 0 & 0 \\
0 & 0 & 0 & 0 & 0 \\
0 & 0 & 0 & \frac{1}{R_{1} C_{1}} & 0 \\
0 & 0 & 0 & 0 & \frac{n}{R_{2} C_{2}}
\end{array}\right] \quad \mathbf{x}=\left[\begin{array}{c}
i_{L d} \\
i_{L q} \\
u_{C 1} \\
u_{C 2}
\end{array}\right] \quad \mathbf{u}=\left[\begin{array}{c}
m_{1 d} \\
m_{2 d} \\
m_{1 q} \\
V_{1} \\
V_{2}
\end{array}\right]
\end{gathered}
$$

\section{CONTROL OBJECTIVE}

As shown in system equations (3), we have three variables on which we may act $\left(m_{1 d}, m_{1 q}, m_{2 d}\right)$ and four state variables, in principle we may only control three of these state variables, leaving a free behavior in the other variable. The control objective is then to assure that each state variable which could be controlled tracks its reference.

\subsection{Under-actuation}

Since we may only arbitrarily control at most three state variables, we will now study whether these input variables we may control these three states. Controllability of general bilinear systems is still an open problem, there exist sufficient conditions for planar bilinear systems (scalar control) (see Koditschek and Narendra (1985) Ayala et al. (2009)), but unfortunately there exits only partial criteria to assure controllability of the systems for the case $n \geq 3$ (see Barras et al. (1996)). Necessary conditions for bilinear controllability systems are shown in Mohler (1991).

In our case the system is not fully actuated, so we can separate it into two parts, one actuated and one non-actuated. This separation will help us to study the controllability of the actuated part.

$$
\begin{array}{r}
\dot{\mathbf{x}}=\left[\begin{array}{c}
\dot{\mathbf{x}}_{a} \\
\dot{\mathbf{x}}_{n a}
\end{array}\right]=\left[\begin{array}{cc}
\mathbf{A}_{a} & \mathbf{A}_{12} \\
\mathbf{A}_{21} & \mathbf{A}_{n a}
\end{array}\right] \cdot\left[\begin{array}{c}
\mathbf{x}_{a} \\
\mathbf{x}_{n a}
\end{array}\right]+ \\
+\left(\sum_{i=1}^{5} u_{i}\left[\begin{array}{cc}
\mathbf{B}_{i_{a}} & \mathbf{B}_{i_{12}} \\
\mathbf{B}_{i_{21}} & \mathbf{B}_{i_{n a}}
\end{array}\right]\right)\left[\begin{array}{c}
\mathbf{x}_{a} \\
\mathbf{x}_{n a}
\end{array}\right]+\left[\begin{array}{c}
\mathbf{C}_{a} \\
\mathbf{C}_{n a}
\end{array}\right] \mathbf{u}
\end{array}
$$

where $\mathbf{x}_{a}$ denotes the actuated states, $\mathbf{x}_{n a}$ denotes the nonactuated state, $\mathbf{A}_{a}$ denotes the actuated part of matrix $\mathbf{A}, \mathbf{A}_{n a}$ denotes the non-actuated part of $\mathbf{A}$ and the same reasoning for $\mathbf{B}$ and $\mathbf{C}$.

Three variables $\left(m_{1 d}, m_{1 q}, m_{2 d}\right)$ are used for controlling three state variables, leaving the last free. The reasoning of the choice of the control variables is the following: since $i_{L q}$ is closely linked with the reactive power consumed by the inductance in the AC step of the converter, and since this power decreases the transmitted active power, it is clearly a variable whose control is important and whose reference in most cases will be to make it as small as possible or even zero. Between the three state variables that remains $\left(i_{L d}, u_{C 1}\right.$ and $\left.u_{C 2}\right)$, we will always chose a voltage as variable to control in order to guarantee the voltage stability of the converter. As we have chosen $u_{C 2}$ as phase reference for PLL, we will chose this variable as control objective, remaining the other two variables to pick one in order to complete the set. We will present results with both control objectives, that means, either we control the trio $\left(i_{L d}, i_{L q}, u_{C 2}\right.$. $)$ or the trio $\left(i_{L q}, u_{C 1}, u_{C 2}\right)$. In both cases we can easily verify the necessary conditions explained above (see Mohler (1991)) for bilinear controllability systems.

\subsection{Bilinear system stability and control law}

Bilinear systems' stability has been studied extensively over the past decades (see Mohler (1991)). In Koditschek and Narendra (1983) the stability of second order bilinear systems under certain conditions is proven. In Landau (1979) a quadratic feedback control $\mathbf{u}(\mathbf{x})$ :

$$
u_{i}=-\alpha\left[\mathbf{B}_{i} \mathbf{x}+\mathbf{c}_{i}\right]^{T} \mathbf{P} \mathbf{x}
$$

with $i=1, \cdots, m$ stabilizes the system presented in (3) in the origin, by means of the quadratic Lyapunov function, $V=\mathbf{x}^{T} \mathbf{P} \mathbf{x}$. Where $\mathbf{P}$ is positive definite and $\alpha>0$.

Indeed, if we derive the Lyapunov function, we obtain

$$
\dot{V}=\mathbf{x}^{T}\left[\mathbf{P A}+\mathbf{A}^{T} \mathbf{P}\right] \mathbf{x}-2 \alpha \sum_{i=1}^{m}\left[\mathbf{x}^{T} \mathbf{P}\left(\mathbf{B}_{i} \mathbf{x}+\mathbf{c}_{i}\right) \mathbf{x}\right]^{2}
$$

where it is assumed that

$$
\left[\begin{array}{c}
\left(\mathbf{B}_{1} \mathbf{x}+\mathbf{c}_{1}\right)^{T} \mathbf{P} \mathbf{x} \\
\vdots \\
\left(\mathbf{B}_{m} \mathbf{x}+\mathbf{c}_{m}\right)^{T} \mathbf{P} \mathbf{x}
\end{array}\right] \neq \mathbf{0}
$$

$\forall \mathbf{x}$ such that $\mathbf{x} \neq \mathbf{0}$ and $\mathbf{x}\left(\mathbf{P A}+\mathbf{A}^{T} \mathbf{P}\right) \mathbf{x} \leq 0$ in order to guarantee $\dot{V} \leq 0$.

Since the system is not fully actuated, and we have separated our system into two parts, we may apply the control strategy presented in 3.2 to the controllable part. Applying the quadratic feedback control explained above and taking into account section 3.1 , we will only act over $i_{L q}, u_{C 1}$ and $u_{C 2}$. So $\mathbf{x}_{a}=\left[i_{L q}, u_{C 1}, u_{C 2}\right]$. If $\overline{\mathbf{x}}_{a}$ is an equilibrium state of the controlled variables corresponding to an input $\overline{\mathbf{u}}$, making the change of variables $\mathbf{x}_{a}=\overline{\mathbf{x}}_{a}+\tilde{\mathbf{x}}_{a}$, and $\mathbf{u}=\overline{\mathbf{u}}+\tilde{\mathbf{u}}$, in order to achieve that the state variables track their references, and taking into consideration that $B(u, x)=\left(\sum_{i=1}^{m} u_{i} \mathbf{B}_{i}\right) \mathbf{x}=\left(\sum_{i=1}^{n} x_{i} \mathbf{H}_{i}\right) \mathbf{u}$, with a suited $H_{i} \in \mathbb{R}^{n \times m}$, we obtain that:

$$
\begin{array}{r}
B\left(\mathbf{u}, \mathbf{x}_{a}\right)=B\left(\overline{\mathbf{u}}+\tilde{\mathbf{u}}, \overline{\mathbf{x}}_{a}+\tilde{\mathbf{x}}_{a}\right)= \\
=B\left(\overline{\mathbf{u}}, \overline{\mathbf{x}}_{a}\right)+B\left(\overline{\mathbf{u}}, \tilde{\mathbf{x}}_{a}\right)+B\left(\tilde{\mathbf{u}}, \overline{\mathbf{x}}_{a}\right)+B\left(\tilde{\mathbf{u}}, \tilde{\mathbf{x}}_{a}\right)
\end{array}
$$

and consequently from (5):

$$
\dot{\tilde{\mathbf{x}}}_{a}=\tilde{\mathbf{A}}_{a} \cdot \tilde{\mathbf{x}}_{a}+B\left(\tilde{\mathbf{u}}, \tilde{\mathbf{x}}_{a}\right)+B_{n a}\left(\tilde{\mathbf{u}}, \mathbf{x}_{n a}\right)+\tilde{\mathbf{C}}_{a} \cdot \overline{\mathbf{u}}
$$
where $B\left(\tilde{\mathbf{u}}, \tilde{\mathbf{x}}_{a}\right)$ and $B_{n a}\left(\tilde{\mathbf{u}}, \mathbf{x}_{n a}\right) \in \mathbb{R}^{5} \rightarrow \mathbb{R}^{3}$ and:

$$
\tilde{\mathbf{A}}_{a}=\left[\mathbf{A}_{a}+\sum_{i=1}^{m} \bar{u}_{i} \mathbf{B}_{a i}\right] \quad \tilde{\mathbf{C}}_{a}=\left[\mathbf{C}_{a}+\sum_{i=1}^{n} x_{a i} \mathbf{H}_{a i}\right] .
$$

Proceeding in analogous form showed above, we are going to prove that the following control (12) stabilizes the system (10) in the desired point given by the references, by means of the quadratic Lyapunov function, $\tilde{V}=\tilde{\mathbf{x}}^{T} \tilde{\mathbf{P}} \tilde{\mathbf{x}}$.

$$
\tilde{u}_{i}=-\alpha\left[\mathbf{B}_{a i} \tilde{\mathbf{x}}_{a}+\tilde{\mathbf{c}}_{a i}+\mathbf{b}_{n a i} \mathbf{x}_{n a}\right]^{T} \tilde{\mathbf{P}} \tilde{\mathbf{x}}_{a}
$$

with $i=1, \cdots, 3$ where $\tilde{\mathbf{c}}_{a i}$ are the columns of the matrix $\tilde{\mathbf{C}}_{a}, \mathbf{b}_{n a i}$ are the columns of matrix $\mathbf{B}_{n a}$, and $\tilde{\mathbf{P}}$ is positive 
definite symmetric matrix of appropriate dimension (in this case the identity matrix of order 3 ).

Proof.- System (10) can be rewritten as

$$
\dot{\tilde{\mathbf{x}}}_{a}=\tilde{\mathbf{A}}_{a} \cdot \tilde{\mathbf{x}}_{a}+\sum_{i=1}^{m}\left(\tilde{u}_{i}\left[\mathbf{B}_{a i} \tilde{\mathbf{x}}_{a}+\tilde{\mathbf{c}}_{a i}+\mathbf{b}_{n a i} \mathbf{x}_{n a}\right]\right)
$$

and taking into account that given column vectors $a$ and $b$ then $a^{T} \tilde{P} b=b^{T} \tilde{P} a$ because $\tilde{P}$ is symmetric. Applying this property:

$\dot{\tilde{V}}=\tilde{\mathbf{x}}_{a}^{T}\left[\tilde{\mathbf{P}} \tilde{\mathbf{A}}_{a}+\tilde{\mathbf{A}}_{a}^{T} \tilde{\mathbf{P}}\right] \tilde{\mathbf{x}}_{a}-2 \alpha \sum_{i=1}^{m}\left[\tilde{\mathbf{x}}_{a}^{T} \tilde{\mathbf{P}}\left(\mathbf{B}_{a i} \tilde{\mathbf{x}}_{a}+\tilde{\mathbf{c}}_{a i}+\mathbf{b}_{n a i} \mathbf{x}_{n a}\right) \tilde{\mathbf{x}}_{a}\right]^{2}$

and $\tilde{\mathbf{A}}_{a}$ in our case has the following form if we control the trio $i_{L d}, i_{L q}, u_{C 2}$

$$
\tilde{\mathbf{A}}_{a}=\left[\begin{array}{ccc}
-\frac{R}{L} & \omega & -\frac{\bar{u}_{2} \sqrt{3}}{L \sqrt{2}} \\
-\omega & -\frac{1}{R_{1} C_{1}} & 0 \\
\frac{n^{2} \bar{u}_{2} \sqrt{3}}{C_{2} \sqrt{2}} & 0 & -\frac{1}{R_{2} C_{2}}
\end{array}\right]
$$

which is Hurwitz $\underset{\sim}{\forall} \bar{u}_{2} \in \mathbb{R}$. Or if we control the trio $i_{L q}, u_{C 1}, u_{C 2}$ then $\tilde{\mathbf{A}}_{a}$ has the form:

$$
\tilde{\mathbf{A}}_{a}=\left[\begin{array}{ccc}
-\frac{R}{L} & \frac{\bar{u}_{3} \sqrt{3}}{L \sqrt{2}} & 0 \\
-\frac{\bar{u}_{3} \sqrt{3}}{C_{1} \sqrt{2}} & -\frac{1}{R_{1} C_{1}} & 0 \\
0 & 0 & -\frac{1}{R_{2} C_{2}}
\end{array}\right]
$$

which is Hurwitz $\forall \bar{u}_{3} \in \mathbb{R}$. So the Lyapunov function $\dot{\tilde{V}}<\lambda \tilde{V}$, with $\lambda$ a positive constant, and consequently the control law shown in (12) stabilizes exponentially the system. (Note that for the other combinations of actuated variables its corresponding $\tilde{\mathbf{A}}_{a}$ are always Hurwitz).

Now remains to study what happens with the nonactuated variable. But in our case, due to it being a first order differential equation with a constant negative value in the coefficient of the state variable, and as we have controlled the remaining states, this free variable is also exponentially stable. $\square$

Theorem 1. The converter described by (3), with arbitrary initial conditions, under control law shown in (12), will exponentially tracks its references.

\subsection{Nonlinear system stability and control law}

As discussed above, bilinear systems are a class of nonlinear systems, but bilinear systems paradoxically have not global stability specified results. Therefore, they are commonly dealt with non-linear control techniques. In this section we will use Lyapunov theory to develop another controller to our system.

If we take a glance on system (3), we observe that the variable $i_{L q}$ may be controlled through the control variable $m_{1 q}$. Something similar happens with the variable $u_{C 2}$, which may be controlled by $m_{2 d}$. So it is clear that both variables can be controlled independently. With respect to the other variables, $i_{L d}$ and $u_{C 1}$, the same reasoning explained in 3.1 is valid, that means, we may only control one of them, and the other has a free behavior. In the section above we have shown a control for the trio $i_{L q}, u_{C 1}$ and $u_{C 2}$. The present section will develop a controller for the trio $i_{L d}, i_{L q}$ and $u_{C 2}$, although one could have chosen the other trio. Let's start with the following equation:

$$
\frac{d}{d t} i_{L q}=-\omega \cdot i_{L d}-\frac{R}{L} \cdot i_{L q}+\frac{m_{1 q} \sqrt{3}}{L \sqrt{2}} \cdot u_{c 1}
$$

Considering the following tracking error

$$
\tilde{i}_{L q}=i_{L q}-\bar{i}_{L q}
$$

with the associated Lyapunov function

$$
Q_{i_{L q}}=\frac{1}{2}\left(i_{L q}-\bar{i}_{L q}\right)^{2}
$$

Its time derivate is:

$$
\dot{Q}_{i_{L q}}=\tilde{i}_{L q} \cdot\left(-\omega \cdot i_{L d}-\frac{R}{L} \cdot i_{L q}+\frac{m_{1 q} \sqrt{3}}{L \sqrt{2}} \cdot u_{c 1}-\dot{\bar{i}}_{L q}\right)
$$

in order to achieve negative derivative of the Lyapunov function:

$$
-\omega \cdot i_{L d}-\frac{R}{L} \cdot i_{L q}+\frac{m_{1 q} \sqrt{3}}{L \sqrt{2}} \cdot u_{c 1}-\dot{\bar{i}}_{L q}=-\alpha_{1} \cdot \tilde{i}_{L q}(21)
$$

with $\alpha_{1}$ positive constant. So, the time derivative becomes:

$$
\dot{Q}_{i_{L q}}=-\alpha_{1} \cdot \tilde{i}_{L q}^{2}
$$

which applying the Lyapunov theory one can guarantee its exponential stability.

The control law for this variable becomes:

$$
m_{1 q}=\frac{L \sqrt{2}}{u_{C 1} \sqrt{3}}\left(\omega \cdot i_{L d}+\frac{R}{L} \cdot i_{L q}+\dot{\bar{i}}_{L q}-\alpha_{1} \cdot \tilde{i}_{L q}\right)
$$

If we apply the same reasoning to the equation ${ }^{1}$ :

$$
\frac{d}{d t} u_{c 2}=\frac{n^{2}}{C_{2}}\left(\sqrt{\frac{3}{2}} m_{2 d} \cdot i_{L d}-\frac{u_{C 2}-V_{2} \cdot n}{n^{2} R_{2}}\right)
$$

and considering the following tracking error

$$
\tilde{u}_{C 2}=u_{C 2}-\bar{u}_{C 2}
$$

with the associated Lyapunov function

$$
Q_{u_{C 2}}=\frac{1}{2}\left(u_{C 2}-\bar{u}_{C 2}\right)^{2}
$$

we obtain the following control law which guaranteed the stability of the variable $u_{C 2}$, where $\alpha_{2}$ is a positive constant:

$$
m_{2 d}=\frac{C_{2} \sqrt{2}}{n^{2} \cdot i_{L d} \sqrt{3}}\left(\frac{u_{C 2}-V_{2} \cdot n}{R_{2} C_{2}}-\dot{\bar{u}}_{C 2}-\alpha_{2} \tilde{u}_{C 2}\right)
$$

At this point, considering that $m_{1 d}$ is the only variable that remains to be defined, we will use it to stabilize $i_{L d}$ proceeding in analogous form to the following equation:

$$
\frac{d}{d t} i_{L d}=-\frac{R}{L} \cdot i_{L d}+\omega \cdot i_{L q}+\frac{m_{1 d} \sqrt{3}}{L \sqrt{2}} \cdot u_{c 1}-\frac{m_{2 d} \sqrt{3}}{L \sqrt{2}} \cdot u_{c 2}
$$

and considering the following tracking error

$$
\tilde{i}_{L d}=i_{L d}-\bar{i}_{L d}
$$

with the associated Lyapunov function

$$
Q_{i_{L d}}=\frac{1}{2}\left(i_{L d}-\bar{i}_{L d}\right)^{2}
$$

\footnotetext{
1 In the following it is important to remark that in practice the three states can never remain equal to zero.
} 
we obtain the following control law which guaranteed the stability of the variable $i_{L d}$, where $\alpha_{3}$ is a positive constant:

$$
m_{1 d}=\frac{L \sqrt{2}}{u_{C 1} \sqrt{3}}\left(\frac{R}{L} i_{L d}-\omega \cdot i_{L q}+\frac{m_{2 d} \sqrt{3}}{L \sqrt{2}} u_{C 2}+\dot{i_{L d}}-\alpha_{3} \tilde{i}_{L d}\right)
$$

Now we will study the behavior of the free state in order to verify if and where it converges. To carry out this task, we will apply the well known theory of zero dynamics (see Isidori (1995) and Chen et al. (2013)). To analyze it, we first divide the state variables (considering the error variables) in two parts:

$$
\begin{array}{r}
\eta=u_{C 1} \\
\xi=\left[\tilde{i}_{L d}, \tilde{i}_{L q}, \tilde{u}_{C 2}\right]^{t}
\end{array}
$$

So the whole system may be considered as in the normal form:

$$
\begin{aligned}
& \dot{\eta}=f_{1}(\eta, \xi, u) \\
& \dot{\xi}=f_{2}(\eta, \xi, u)
\end{aligned}
$$

with

$$
u=f_{3}(\eta, \xi)
$$

given the equations (23), (27) and (31).

So given $u$ such that $\xi \rightarrow \bar{\xi}(\bar{\xi}$ could be zero or not) then the behavior of the system (33) is governed by the differential equation:

$$
\dot{\eta}=f_{1}(\eta, \bar{\xi})
$$

If we apply this result to system (3), the behavior of the free variable $u_{C 1}=\eta$ could be written as:

$$
\dot{u}_{C 1}=\frac{k_{1}}{u_{C 1}}+k_{2} \cdot u_{C 1}+k_{3}
$$

where

$$
\begin{gathered}
k_{1}=-\frac{1}{C_{1}}\left(R\left(\bar{i}_{L d}^{2}+\bar{i}_{L q}^{2}\right)+\frac{1}{n^{2}}\left(\frac{\bar{u}_{C 2}-V_{2} \cdot n}{R_{2}}\right) \bar{u}_{C 2}\right) \\
k_{2}=-\frac{1}{C_{1} R_{1}}, \quad k_{3}=\frac{V_{1}}{C_{1} R_{1}}
\end{gathered}
$$

If we study the behavior of equation (36), calling $f\left(u_{C 1}\right)=$ $\frac{k_{1}}{u_{C 1}}+k_{2} \cdot u_{C 1}+k_{3}$, and taking into account that in steady state by energy balance it is always true that:

$$
\frac{\bar{u}_{C 1}-V_{1}}{R_{1}} \bar{u}_{C 1}=R\left(\bar{i}_{L d}^{2}+\bar{i}_{L q}^{2}\right)+\frac{1}{n^{2}}\left(\frac{\bar{u}_{C 2}-V_{2} \cdot n}{R_{2}}\right) \bar{u}_{C 2}
$$

then the derivate of $f$ is always negative and consequently equation (36) is globally exponentially stable, so the whole system is stabilized by the control law $u=f_{3}(\eta, \xi)$ given by equations (23), (27) and (31). We could obtain an analogous result, if we had chosen $u_{C 1}$ instead of $i_{L d}$ proceeding in a similar form.

Theorem 2. The converter described by (3) under control laws (23), (27) and (31) (with $\alpha_{1}, \alpha_{2}$ and $\alpha_{3}$ positive constants) is globally exponentially stabilized to an equilibrium point given by its references.

The proof of theorem 2 is composed by the steps of the current subsection.

\section{SIMULATIONS}

The controllers studied in the previous sections are tested in MATLAB-Simulink.

\subsection{Bilinear control}

In this section it is shown the simulations of control for the trio $i_{L q}, u_{C 1}$ and $u_{C 2}$. The parameter values of the simulated example are presented in table 1 .

Table 1. Simulation values.

\begin{tabular}{|c|c|c|}
\hline $\mathrm{n}$ & Transformer ratio & $11 / 100$ \\
\hline$R_{\text {total }-i}$ & Total resistance branch $i$ & $0.022 \Omega$ \\
\hline$R_{1}$ & Resistance source 1 & $0.001 \Omega$ \\
\hline$R_{2}$ & Resistance source 2 & $0.1 \Omega$ \\
\hline$V_{1}$ & Input voltage & $1000 \mathrm{~V}$ \\
\hline$V_{2}$ & Output voltage & $10000 \mathrm{~V}$ \\
\hline$L_{i}$ & Inductance branch $i$ & $0.01 \mathrm{H}$ \\
\hline$C_{1}$ & Input capacitor & $1 \mathrm{mF}$ \\
\hline$C_{2}$ & Output capacitor & $20 \mu \mathrm{F}$ \\
\hline$f$ & Switching frequency & $1000 \mathrm{~Hz}$ \\
\hline
\end{tabular}

In figure 3 the behavior of $i_{L q}$ is shown. We observe as the variable tracks its references (100 A until $0.4 s$ and $85 \mathrm{~A}$ for the rest) satisfactorily.

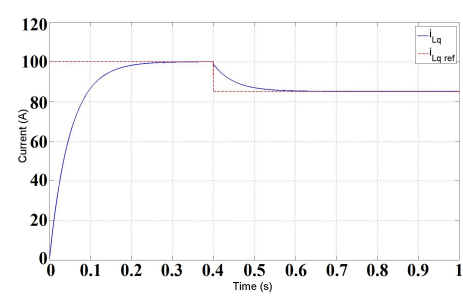

Fig. 3. $i_{L q}$ behavior (bilinear control).

In figure 4 the behavior of $u_{C 1}$ and $u_{C 2}$ are shown. We observe as the $u_{C 1}$ tracks its reference $(990 \mathrm{~V}$ until 0.5 $s$ and $980 \mathrm{~V}$ for the rest) in an appropriate way, where the voltage has a small oscillation band, caused by the size of the capacitors. For $u_{C 2}$, we observe as the variable tracks its references $(10015 \mathrm{~V}$ until $0.8 s$ and $10005 \mathrm{~V}$ for the remaining) satisfactorily. Observing figure 4 it is clear that the power direction flows from left side to right side of the converter, so $i_{L d}$, the free variable, must be positive. This is in according with the simulations shows in figure 5

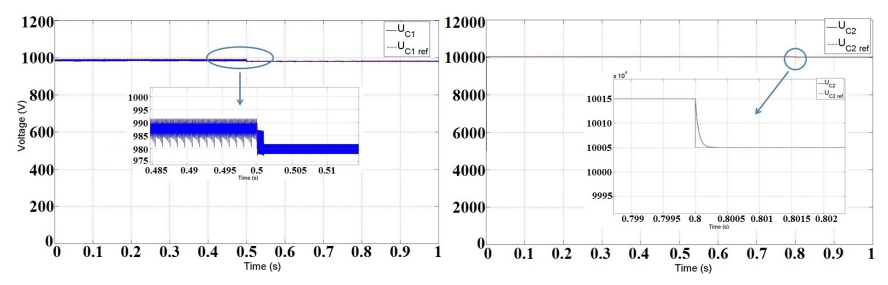

Fig. 4. a) $u_{C 1}$ b) $u_{C 2}$ behavior (bilinear control).

\subsection{Nonlinear control}

In this section it is shown the simulations for the control of the trio $i_{L d}, i_{L q}$ and $u_{C 2}$, leaving $u_{C 1}$ as free variable. The parameter values of the simulated example are the same as above. 


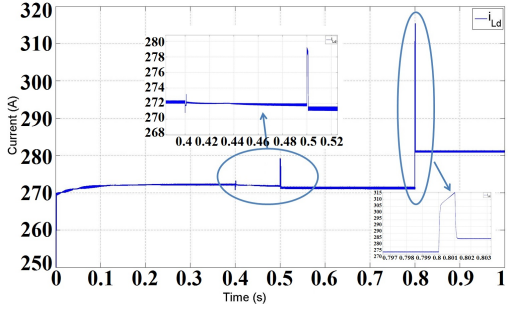

Fig. 5. $i_{L d}$ behavior (bilinear control).

In figure 6 the behavior of $i_{L d}$ and $i_{L q}$ are shown. We observe as in figure 6 a the variable tracks its references satisfactorily(-200 A until $0.8 s$ and -250 A for the remaining). In figure $6 \mathrm{~b}$ the variable tracks its references satisfactorily(-15 A until $1.2 s$ and $-45 \mathrm{~A}$ for the rest).

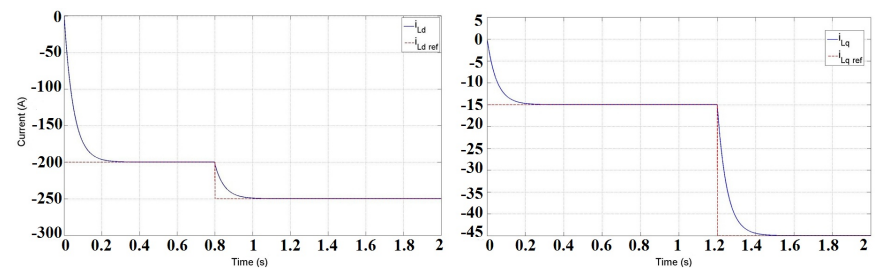

Fig. 6. a) $i_{L d}$ b) $i_{L q}$ behavior (nonlinear control).

In figure $7 \mathrm{~b}$ the behavior of $u_{C 2}$ is shown. We observe as the variable tracks its references $(9990 \mathrm{~V}$ until $1.6 \mathrm{~s}$ and $9995 \mathrm{~V}$ for the remaining) satisfactorily. We observe that due to the current has negative value and the voltage in capacitor $C_{2}$ is less than $V_{2}=10000 \mathrm{~V}$, the power direction flows from right side to left side of the converter. So $u_{C 1}$ (figure $7 \mathrm{a}$ ), the free variable, must be greater than $V_{1}=1000 \mathrm{~V}$. We can check it showing figure $7 \mathrm{a}$.
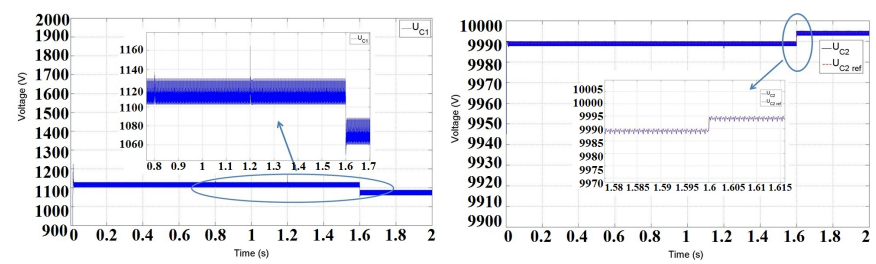

Fig. 7. a) $u_{C 1}$ b) $u_{C 2}$ behavior (nonlinear control).

\section{COMPARISON}

In view of the results, we can say that both controllers provide interesting results, and they are able to correctly stabilize the system. Due to its simplicity, bilinear control is easier to implement, and may be preferable for real applications. On the other hand, fully nonlinear approaches have a deeper established theory, with broader tools. For this reason it can be more interesting for future works.

\section{CONCLUSIONS}

The main objective of this paper is to provide a control for a DC/DC converter used in a multi-terminal DC grid in order to integrate renewable energy. In order to solve the problem, two viewpoints have been addressed and compared. Due to the intrinsic nature of this type of converters, the problem leads to a bilinear approach in a natural way, so a solution supported by bilinear theory has been presented. Due to the system being not completely actuated, a solution for the actuated subsystem has been developed. In a second step a nonlinear control has been developed. A detailed stability analysis of the zero dynamics shows that our system is exponentially stable. Both control algorithms provide suitable results in simulations, so both will be considered for subsequent test bed experimentations.

\section{REFERENCES}

Arrillaga, J., Liu, Y., and Watson, N. (2007). Flexible Power Transmission. The HVDC Options. John Wiley \& Sons Ltd.

Asplund, G. (2007). Ultra high voltaje trasmission. $A B B$ review.

Ayala, V., Cruz, E., and Kliemann, W. (2009). Controllability of bilinear systems on the projective space. Computer and Mathematics with Applications.

Barragán-Villarejo, M., Venkataramanan, G., MancillaDavid, F., Maza-Ortega, J., and Goómez-Expósito, A. (2012). Dynamic modelling and control of a shunt-series power flow controller based on ac-link. IET Generation, Transmission \& Distribution, 792-802.

Barras, J., Goncalves, J., do Rocio, O., and Martín, L.S. (1996). Controllability of two-dimensional bilinear systems. Proyeciones, 111-139.

Chen, Y., Dai, J., Damm, G., and Lamnabhi-Lagarrigue, F. (2013). Analysis of a control strategy for a multiterminal vsc-hvdc system. ECC.

Elliot, D. (2009). Bilinear Control Systems. Springer.

G.Ortiz, Biela, J., Bortis, D., and Kolar, J. (2010). 1 mw, $20 \mathrm{khz}$, isolated, $1.2-12 \mathrm{kv}$ bidirectional dc-dc renawable converter for energy applications. International Power Electronics Conference, IEEE.

Isidori, A. (1995). Nonlinear control systems. Springer Verlag.

Jiménez, M., Benchaib, A., Alou, P., and Damm, G. (2013). Dc transformer for dc/dc connection in hvdc network. 15th European Conference on Power Electronics and Applications.

Khalil, H.K. (2002). Nonlinear systems. Prentice Hall.

Koditschek, D. and Narendra, K. (1983). Stabilizity of $2^{n} d$ order bilinear systems. IEEE Transactions on Automatic control.

Koditschek, D. and Narendra, K. (1985). The controllability of planar bilinear systems. IEEE Transactions on Automatic control, 87-89.

Landau, I. (1979). On the optimal regulator problem and stabilization of bilinear systems. Laboratoire d'Automatique (CNRS).

Mendel, S., Vogel, C., and Dalt, N.D. (2009). IEEE Transactions on Circuits and Systems II: Express Briefs, $56(11), 860-864$.

Mohler, R. (1973). Bilinear Control Processes. Academic Press.

Mohler, R. (1991). Nonlinear systems. Applications to Bilinear Control. Printence Hall.

Park, R. (1929). Two reaction theory of synchronous machines.

Sira-Ramirez, H., Garcia-Esteban, M., and Zinober, A. (1994). Dynamical adaptive pulse-width-modulation control of dc-to-dc power converters: a backstepping approach. Journal of control, 205-222. 